\title{
Haemorrhagic cystitis due to cytomegalovirus in a patient with AIDS
}

\author{
Winston P. R. Padayachee ${ }^{1}$, Sanjay Sadhwani ${ }^{1}$, Sean W. Doherty ${ }^{1}$, Alain Mwamba Mukendi ${ }^{*}$ (D), \\ Eunice Van den Berg ${ }^{2}$ and Adam R. Botha ${ }^{2}$
}

\begin{abstract}
Background: Cytomegalovirus-related infections are commonly seen in immunocompromised patients. However, haemorrhagic cystitis is an exceptionally rare associated manifestation. We present an unusual case of cytomegalovirus-related haemorrhagic cystitis in a patient with acquired immune deficiency syndrome (AIDS).

Case presentation: A 33-year-old HIV-positive female presented with acute gross haematuria and suprapubic pain. Cystoscopy revealed features suggestive of haemorrhagic cystitis with clots in the bladder and an ulcerative lesion on the left lateral wall which was biopsied. Histology demonstrated the presence of enlarged cells containing eosinophilic intranuclear and ill-defined amphophilic intracytoplasmic inclusions. Immunohistochemistry was positive for cytomegalovirus.

Conclusion: This case appears to be the first of its kind reported in South Africa highlighting the importance of considering cytomegalovirus as a potential infectious aetiology in AIDS patients with haematuria. Bladder irrigation with normal saline with or without clot evacuation combined with Ganciclovir are associated with good outcome.
\end{abstract}

Keywords: Cytomegalovirus, Haemorrhagic cystitis, AIDS

\section{Background}

Symptomatic cytomegalovirus (CMV) infection is common in immunocompromised individuals and may produce a diverse spectrum of disease. Whilst gastrointestinal, ocular and neurologic manifestations are well described in the setting of AIDS, urinary tract manifestations are rare and are reported predominantly following stem cell transplants [1]. We present an unusual case of CMV associated haemorrhagic cystitis in an individual infected with acquired immune deficiency syndrome (AIDS).

\subsection{Case presentation}

A 33-year-old HIV-positive female presented with a history of acute onset haematuria and suprapubic pain.

\footnotetext{
*Correspondence: alainmwamba2006@yahoo.fr

${ }^{1}$ Department of Urology, Chris Hani Baragwanath Academic Hospital,

University of Witwatersrand, Johannesburg, South Africa

Full list of author information is available at the end of the article
}

She had defaulted on her anti-retroviral regimen 3 years earlier, due to drug intolerance. Her current absolute CD4 count was 20 cells $/ \mu \mathrm{L}$ and her HIV viral load was 2,110,000 copies/ml. Physical examination revealed total gross haematuria with urinary retention due to clots. There was no necroturia.

Urine microscopy revealed the presence of numerous erythrocytes but no red blood cell casts, leucocytes, acid-fast bacilli, parasites or bacterial growth. Contrast enhanced abdominal computed tomography revealed a non-enhancing filling defect in the bladder. No other urinary tract abnormalities were detected. Serological markers for inflammation were not raised. The coagulation profile was unremarkable and her renal function was normal.

Treatment was commenced with analgesia and continuous saline irrigation following a bedside bladder washout. After $72 \mathrm{~h}$ of bladder irrigation, her urine was macroscopically clear. Cystoscopy demonstrated oedematous and haemorrhagic mucosa, with residual 
clots in the bladder and an ulcerative lesion on the left lateral wall which was biopsied (Fig. 1).

A provisional diagnosis of haemorrhagic cystitis of unknown aetiology was made. Three weeks after the initial presentation, the patient was readmitted with a recurrence of haematuria. She was once again commenced on saline bladder irrigation.

Histology from the biopsy demonstrated ulceration with underlying granulation tissue, the presence of a significant mixed inflammatory cell infiltrate within the lamina propria consisting of lymphocytes, histiocytes and eosinophils. Focal enlarged cells demonstrating viral cytopathy were identified, with ill-defined amphophilic intracytoplasmic inclusions and large nuclei. A chronic active cystitis was present with focal cells showing viral cytopathy (Fig. 2). Immunohistochemistry was positive for cytomegalovirus (Fig. 3).

Serology for CMV revealed IgG positive, IgM negative, and CMV viral load $1165 \mathrm{IU} / \mathrm{ml}$. Screening for CMV retinitis revealed no abnormalities. The decision was made to treat with Ganciclovir $5 \mathrm{mg} 12$ hourly intravenously for 14 days. Symptoms improved following bladder irrigation and antiviral therapy with no recurrence of haematuria at 6 months. Follow-up cystoscopy revealed a resolution of the mucosal ulceration with a small residual posterior wall oedema and punctate mucosal haemorrhages.

The patient was referred for re-initiation of antiretroviral treatment, with a reduction in the HIV viral load to 2130 copies/ml after 6 weeks of treatment.

\section{Discussion}

Our patient report outlines a rare case of CMV haemorrhagic cystitis not associated with transplant, but rather with HIV/AIDS. This case appears to be the first of its kind reported in South Africa. With 4 previously documented cases, a review of available literature suggests

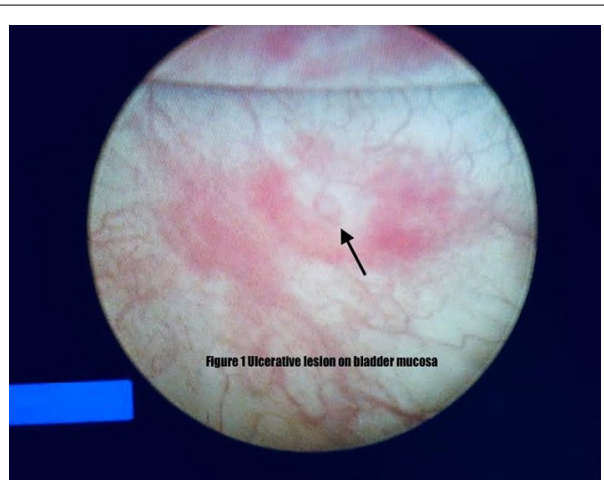

Fig. 1 Cystoscopy image showing an ulcerative lesion surrounded by areas of inflammation of the mucosa

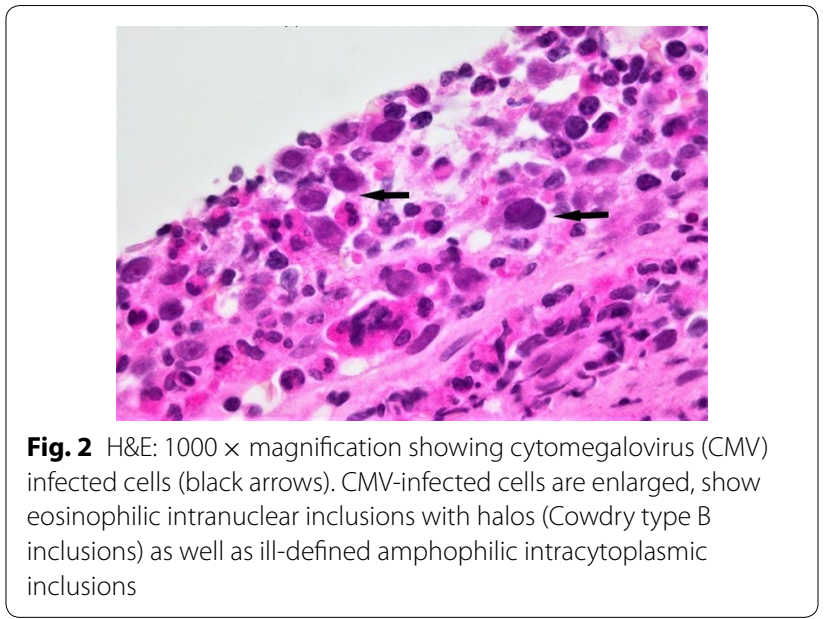

that cystitis as a sole clinical manifestation of CMV infection in a patient with AIDS is rare. In all previously described cases, CMV was diagnosed on bladder biopsy in patients with symptoms such as bladder pain or haematuria. In at least one case, endoscopy revealed macroscopically normal bladder mucosa. A case of more complicated cystitis with resultant bladder perforation and concomitant ureteritis has also been described. At least two of the patients received antiviral therapy, which was followed by symptom resolution [1-4].

\subsection{Haemorrhagic cystitis}

Haemorrhagic cystitis is defined as diffuse inflammation of the bladder resulting in mucosal bleeding [5]. Droller et al. described a grading system for the classification of haemorrhagic cystitis.

0-No symptoms of bladder irritability, no haematuria.

1-Microscopic haematuria

2-Macroscopic haematuria

3-Macroscopic haematuria with small clots

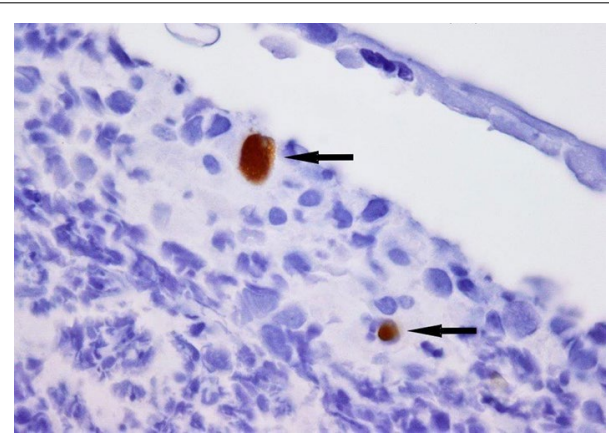

Fig. 3 H\&E: $1000 \times$ magnification showing positive staining of CMV-infected cells with CMV immunochemistry (black arrows) 
4-Massive macroscopic haematuria requiring instrumentation for clots and/or clot retention [6].

Haemorrhagic cystitis is usually managed by a urologist, particularly when macroscopic haematuria with clots is present. Patients may require haemodynamic stabilisation and/or transfusion, as well as empiric antimicrobial therapy when an infective cause is suspected. Coagulopathy should be excluded. Evacuation of clots may be performed via a three-way Foley urethral catheter or via cystoscopy in severe cases. During cystoscopy, fulguration of vascular lesions may be performed [5]. Laser or argon beam ablation of bleeding vessels at the time of cystoscopy has also been described [7]. Following this, saline irrigation is commenced to prevent clot retention and the complications of obstructive uropathy. Should the haematuria persist despite these conventional interventions, a number of adjunctive intravesical, systemic and surgical therapies may be considered for haemostasis [5].

Intravesical alum therapy (aluminium ammonium sulphate or aluminium potassium sulphate) has been described for persistent haematuria due to haemorrhagic cystitis. A $1 \%$ solution in sterile water is typically described, although normal saline has also been used. The solution is run as bladder irrigation at approximately 250-300 $\mathrm{ml} / \mathrm{h}$. Aluminium salts have astringent properties within the bladder, resulting in vasoconstriction and reduced capillary permeability by means of protein precipitation. In one series, response to alum irrigation was observed in approximately $60 \%$ of patients. The most frequent adverse effect is bladder spasm. Aluminium toxicity is rare, but is of particular concern in patients with existing renal impairment, which may limit the excretion of serum aluminium [8]. As a practical, fast-acting and well-tolerated therapy, intravesical alum has been recommended as a first line therapy for haemorrhagic cystitis in inpatients with normal renal function who do not respond to conventional bladder irrigation [9].

Hyaluronic acid (HA), a mucopolysaccharide with immunomodulatory properties, has been recommended as an intravesical therapy. Instillation into the bladder is thought to aid in the repair of the glycoaminoglycan layer, an important urothelial defence mechanism against toxins and bacterial adherence $[5,7,9]$. Six doses of $40 \mathrm{mg}$ hyaluronic acid over 3 months, each retained within the bladder for $20 \mathrm{~min}$, have been found to be at least as efficacious as hyperbaric oxygen therapy for reducing bleeding in haemorrhagic cystitis and adverse effects are uncommon [10]. Its use, however, is limited to stable outpatients with haematuria due to its slow onset of action. As an established therapy for painful bladder syndrome, HA is particularly useful in patients with radiation cystitis who report haematuria as well as bothersome lower urinary tract symptoms [7].

Other intravesical agents have been described for treatment of haemorrhagic cystitis, including e-aminocaprioc acid, silver nitrate, fibrin glue, phenol and prostaglandins. The evidence for these therapies is mostly described within small case series and as such they have yet to be recommended for routine use $[5,7,9]$.

Hyperbaric oxygen therapy (HBOT) has been shown to be safe and effective in the treatment of haemorrhagic cystitis, particularly in the setting of radiation-induced cystitis. The inhalation of $100 \% \mathrm{O}_{2}$ at 1.4-3 atmospheres results in tissue hyperoxygenation which induces neovascularisation, promotes healthy granulation tissue deposition and causes vasoconstriction. Up to $87.5 \%$ of patients experience resolution of bleeding. The most frequently reported complications are otitis and visual disturbances related to barotrauma. HBOT has been recommended as a first-line therapy for persistent bleeding due to haemorrhagic cystitis and may be applied in an inpatient or outpatient setting, but its use may be limited by access to the resources and skills required for administration $[7,9]$.

Systemic treatment with oral sodium pentosanpolysulphate (SPP) may reduce bleeding in haemorrhagic cystitis. Its mechanism of action is comparable to HA, and similarly, it may be beneficial for patients with lower urinary tract symptoms. A few case series' have demonstrated it to be a practical treatment, with the advantages of oral administration and few adverse effects [7]. An initial dose of $100 \mathrm{mg}$ three times a day followed by a reduced maintenance dose of $100 \mathrm{mg}$ daily resulted in complete or partial resolution of haematuria in approximately $50 \%$ of patients in a single series [11]. SPP has a slow onset of action (up to 8 weeks), and its use is therefore most appropriate in stable outpatients with recurrent haematuria [7].

At present there is insufficient evidence to recommend routine administration of systemic tranexamic acid in the setting of haemorrhagic cystitis. Clinicians should also be aware of the concern of clot formation within the urinary tract [9]. WF10 is another experimental intravenous treatment which has been studied for use in haemorrhagic cystitis. It is thought to reduce urothelial inflammation through its actions on macrophages, T-lymphocytes and natural killer cells [7].

Patients with refractory haematuria, who fail to respond to the aforementioned therapies, may require more drastic intervention, particularly if the bleeding becomes life-threatening.

Intravesical formalin instillation is a rapid and effective therapy which is believed to cause occlusion of the urothelial microvasculature by protein precipitation. 
Morbidity and mortality associated with formalin use is proportional to the concentration used. The exclusion of vesico-ureteric reflux is essential prior to administration to avoid secondary ureteric obstruction. Lower concentrations of $1-2 \%$, retained within the bladder for $15 \mathrm{~min}$ under general or regional anaesthetic, are best tolerated and associated with resolution of haematuria in approximately $70 \%$ of patients. Intravesical formalin should be reserved for patients who fail to respond to conventional non-invasive therapies and clinicians should be aware of the need to monitor for potential complications following administration $[5,7,9]$.

Arterial embolization has been recommended as a treatment for refractory haematuria from various aetiologies, including haemorrhagic cystitis. The procedure does not require general anaesthesia and has a response rate of $88-100 \%$. The most common complication is gluteal pain due to superior gluteal artery occlusion. However, superselective embolization using microparticles reduces this and other ischaemic complications [5, 9].

Rarely, refractory haemorrhagic cystitis may warrant surgical intervention. Urinary diversion alone may be performed to reduce the exposure of bleeding surfaces to urokinase. However, cystectomy should also be considered for haemostasis particularly in patients who are able to tolerate the procedure $[5,9]$. Cystectomy with urinary diversion for bleeding is a last resort, as the procedure is associated with a $42 \%$ incidence of severe complications and up to $16 \%$ of patients may not survive beyond 90 days [12].

Once the cause of bleeding has been identified, additional disease-specific therapy may be required.

\subsection{Cytomegalovirus cystitis}

A comprehensive history and physical examination in conjunction with urinalysis, imaging and endoscopic evaluation may differentiate haemorrhagic cystitis from other causes of haematuria such as malignancy, stones, benign prostatic hyperplasia, trauma and intrinsic glomerular disease. Common non-infectious causes of cystitis include radiation, chemotherapy (especially cyclophosphamide and ifosfamide), environmental toxins and interstitial cystitis. Systemic diseases such as rheumatoid arthritis, amyloidosis and Crohn's disease may also cause cystitis. Whilst infectious cystitis refers commonly to bacterial infection, it may also refer to viral, fungal, schistosomal or mycobacterial disease [5].

Viral cystitis is usually described in patients post-haemopoetic cell transplantation. In particular, BK polyomavirus and adenovirus have been identified as causing haemorrhagic cystitis in this setting [13]. Cases involving cytomegalovirus, JC polyomavirus or human herpesvirus- 6 are encountered less frequently [5].

Cytomegalovirus (CMV) is a betaherpesvirus that commonly infects humans. Primary infection is usually asymptomatic or may produce a syndrome resembling infectious mononucleosis. Thereafter the virus remains latent. Host T cell-mediated immunity controls viral replication, with immunocompetent hosts generally remaining asymptomatic. CMV disease, therefore, occurs largely in the setting of immunological suppression or immaturity $[14,15]$.

Disease in these immunocompromised hosts may be due to primary infection but more commonly represents reactivation. In post-transplant patients, the risk is greatest in recipients who are seropositive for CMV, particularly when receiving grafts from a seronegative donor [16]. In HIV-positive patients, symptomatic disease typically occurs in those with $\mathrm{CD}^{+}$cell counts below $100 \mu \mathrm{l}$ and/or HIV viral loads of $>100,000$ copies/ml [17, 18]. Retinal, gastrointestinal and central nervous system CMV disease is well described in immunosuppressed solid organ or haemopoetic cell transplant recipients and also in individuals with AIDS. Reports of urinary manifestations, however, including ureteritis and cystitis, are largely confined to individuals post-haemopoetic cell transplant $[19,20]$.

The diagnosis of CMV disease may be challenging. Viral replication and shedding may occur without producing symptomatic disease. Laboratory tests have a limited value in proving end-organ disease [17].

In this case, the patient's positive IgG with a negative IgM may be suggestive of reactivation disease. However, serology for suspected CMV disease in immunocompromised individuals has limited diagnostic value on its own [18, 21].

CMV viral loads, obtained using quantitative PCR, are commonly used to predict disease and initiate preemptive antiviral therapy in asymptomatic, seropositive patients. Patients with symptoms, however, may be treated at lower viral loads. Results are difficult to interpret because of concerns regarding standardisation across sample types and different assays. There is also a lack of consensus on the threshold values for treatment, particularly in AIDS patients [18, 21].

CMV cystitis is diagnosed when the virus is identified (by culture, immunohistochemical analysis, or in situ hybridisation) and histologic features of inflammation and CMV infection are present on bladder biopsy [22]. In fact, histopathology remains the gold standard for diagnosing all CMV disease and organ involvement. Characteristic features include cellular and nuclear enlargement as well as the presence of cytoplasmic inclusions-aggregates of CMV nucleoproteins as a 
result of viral replication [23]. Cystoscopy and biopsy, therefore, is a key component in the evaluation of undiagnosed haemorrhagic cystitis in immunocompromised patients. Some authors suggest that deeper biopsies, obtained using a resectoscope, may be required for diagnosis [4].

Once symptomatic disease is diagnosed, Ganciclovir is the drug of choice for the treatment of CMV. Patients should be monitored for neutropaenia and symptom improvement [21]. Cidofovir is an alternative reserved for Ganciclovir-refractory disease, although nephrotoxicity may be a concern [24].

\section{Conclusion}

Haemorrhagic cystitis remains a challenging condition for urologists to manage. This case highlights the importance of considering CMV as a potential infectious aetiology in AIDS patients with haematuria more especially when they are not on HAART. Under these circumstances, bladder biopsy may play an important role in the evaluation of undiagnosed lower urinary tract symptoms or haemorrhagic cystitis. Multisystem disease may not always be present and cystoscopic features are not pathognomic, and therefore, a low threshold for biopsy should be maintained. Bladder irrigation with normal saline with or without clot evacuation combined with Ganciclovir is effective in the resolution of symptoms and the prevention of complications in these patients.

\section{Abbreviations}

AIDS: acquired immune deficiency syndrome; BKV: BK virus; CD4: cluster of differentiation 4; CMV: cytomegalovirus; HIV: human immunodeficiency virus; HA: hyaluronic acid; HBOT: hyperbaric oxygen therapy; SPP: sodium pentosanpolysulphate; IgG: immunoglobulin G; IgM: immunoglobulin M; PCR: polymerase chain reaction.

\section{Acknowledgements}

None.

\section{Authors' contributions}

WPRP conceived and designed the study, wrote the manuscript, revised it for critical content. SS wrote the manuscript and obtained consent from the patient. SWD revised manuscript for critical content. AMM obtained ethical approval, revised manuscript for critical content, wrote the abstract and prepare manuscript for submission. EVDB provided histopathological content and slides. ARB provided histological content and slides. They all approved the final version of the manuscript.

\section{Funding}

This study had no funding from any resource.

\section{Competing interests}

The authors declare that they have no competing interests.

\section{Availability of data and materials}

The datasets used during the current study are available from the corresponding author on reasonable request.

\section{Consent for publication}

Written informed consent was obtained from the patient for publication of this manuscript and accompanying pictures. A copy of the written consent is available for review by the Editor-in-Chief of this journal.

\section{Ethics Approval and Consent to Participate}

This study was approved by the Wits- Human Research Ethics Committee, Reference Number of approval: M1910100. The patient included in this study gave written informed consent to participate in this research.

\section{Author details}

${ }^{1}$ Department of Urology, Chris Hani Baragwanath Academic Hospital, University of Witwatersrand, Johannesburg, South Africa. ${ }^{2}$ Department of Anatomical Pathology, University of Witwatersrand, Johannesburg, South Africa.

Received: 11 December 2019 Accepted: 10 June 2020

Published online: 24 July 2020

\section{References}

1. Benson MC, Kaplan MS, O'Toole K et al (1988) A report of cytomegalovirus cystitis and a review of other genitourinary manifestations of the acquired immune deficiency syndrome. J Urol 140(1):153-154. https:// doi.org/10.1016/s0022-5347(17)41515-1

2. Lucas SB, Parr DC, Wright E et al (1989) AIDS presenting as cytomegalovirus cystitis. Br J Urol 64(4):429-430. https://doi.org/10.1111/j.1464410x.1989.tb06060.x

3. Whitaker JA, Jacob JT, Little JV et al (2008) Cytomegalovirus cystitis with bladder wall dehiscence in a patient with AIDS. AIDS 22(6):795-796. https ://doi.org/10.1097/qad.0b013e3282f56103

4. Goncalvez J, Villez JP, Nouveau J et al (1995) Cytomegalovirus cystitis and AIDS. The value of deep bladder biopsy. Apropos of a case. Prog Urol 5:407-409

5. Dorairajan L, Manikandan R, Kumar S (2010) Hemorrhagic cystitis: a challenge to the urologist. Indian J Urol 26(2):159. https://doi. org/10.4103/0970-1591.65380

6. Droller MJ, Saral R, Santos G (1982) Prevention of cyclophosphamideinduced hemorrhagic cystitis. Urology 20(3):256-258. https://doi. org/10.1016/0090-4295(82)90633-1

7. Pascoe C, Duncan C, Lamb BW, Davis NF, Lynch TH, Murphy DG, Lawrentschuk N (2019) Current management of radiation cystitis: a review and practical guide to clinical management. BJU Int 123:585-594. https://doi. org/10.1111/bju.14516

8. Westerman M, Boorjian S, Linder B (2016) Safety and efficacy of intravesical alum for intractable hemorrhagic cystitis: a contemporary evaluation. Int Braz Urol 42(6):1144-1149. https://doi.org/10.1590/\$1677-5538. IBJU.2015.0588

9. Goucher G, Saad F, Lukka H, Kapoor A (2019) Canadian urological association best practice report: diagnosis and management of radiationinduced hemorrhagic cystitis. Can Urol Assoc J 13(2):15-23. https://doi. org/10.5489/cuaj.5788

10. Shao Y, Lu G, Shen Z (2011) Comparison of intravesical hyaluronic acid instillation and hyperbaric oxygen in the treatment of radiation-induced hemorrhagic cystitis. BJU Int 109(5):691-694. https://doi.org/10.1111/ j.1464-410X.2011.10550.x

11. Sandhu S, Goldstraw M, Woodhouse C (2004) The management of haemorrhagic cystitis with sodium pentosan polysulphate. BJU Int 94(6):845-847. https://doi.org/10.1111/j.1464-410X.2004.05044.x

12. Linder B, Tarrell R, Boorjian S (2014) Cystectomy for refractory hemorrhagic cystitis: contemporary etiology. Presentation and outcomes. J Urol 192(6):1687-1692. https://doi.org/10.1016/j.juro.2014.06.030

13. Paduch DA (2007) Viral lower urinary tract infections. Curr Urol Rep 8(4):324-335. https://doi.org/10.1007/s11934-007-0080-y

14. Vancikova Z, Dvorak P (2001) Cytomegalovirus Infection in Immunocompetent and Immunocompromised Individuals: a review. Curr Drug Target Immune Endocr Metab Disorders 1(2):179-187. https://doi. org/10.2174/1568005310101020179

15. Gandhi MK, Khanna R (2004) Human cytomegalovirus: clinical aspects, immune regulation, and emerging treatments. Lancet Infect Dis 4(12):725-738. https://doi.org/10.1016/s1473-3099(04)01202-2 
16. Ljungman $P$ (2014) The role of cytomegalovirus serostatus on outcome of hematopoietic stem cell transplantation. Curr Opin Hematol 21(6):466469. https://doi.org/10.1097/moh.0000000000000085

17. De la Hoz RE, Stephens G, Sherlock C (2002) Diagnosis and treatment approaches of CMV infections in adult patients. J Clin Virol 25:1-12. https ://doi.org/10.1016/s1386-6532(02)00091-4

18. Ross S, Novak Z, Pati S, Boppana S (2011) Overview of the diagnosis of cytomegalovirus infection. Infect Disorders Drug Targets 11(5):466-474. https://doi.org/10.2174/187152611797636703

19. Ho M (2007) The history of cytomegalovirus and its diseases. Med Microbiol Immunol 197(2):65-73. https://doi.org/10.1007/s00430-007-0066-x

20. Han T-T, Xu L-P, Liu D-H et al (2013) Cytomegalovirus is a potential risk factor for late-onset hemorrhagic cystitis following allogeneic hematopoietic stem cell transplantation. Am J Hematol 89(1):55-61. https://doi. org/10.1002/ajh.23584

21. Mayaphi SH, Brauer M, Morobadi DM et al (2014) Cytomegalovirus viral load kinetics in patients with HIV/AIDS admitted to a medical intensive care unit: a case for pre-emptive therapy. PLoS ONE 9(4):e93702. https:// doi.org/10.1371/journal.pone.0093702

22. Ljungman P, Griffiths P, Paya C (2002) Definitions of cytomegalovirus infection and disease in transplant recipients. Clin Infect Dis 34(8):10941097. https://doi.org/10.1086/339329

23. Razonable RR, Hayden RT (2013) Clinical utility of viral load in management of cytomegalovirus infection after solid organ transplantation. Clin Microbiol Rev 26(4):703-727. https://doi.org/10.1128/cmr.00015-13

24. Ljungman $P$ (2001) Cidofovir for cytomegalovirus infection and disease in allogeneic stem cell transplant recipients. Blood 97(2):388-392. https:// doi.org/10.1182/blood.v97.2.388

\section{Publisher's Note}

Springer Nature remains neutral with regard to jurisdictional claims in published maps and institutional affiliations.

\section{Submit your manuscript to a SpringerOpen ${ }^{\circ}$ journal and benefit from:}

- Convenient online submission

- Rigorous peer review

- Open access: articles freely available online

- High visibility within the field

- Retaining the copyright to your article

Submit your next manuscript at $\mathbf{s p r i n g e r o p e n . c o m ~}$ 